\title{
Bases Teóricas para a Implementação do Aprendizado Orientado por Problemas na Residência Médica em Anestesiologia *
}

\section{Theoretical Basis for the Implementation of Problem-Oriented Learning in Anesthesiology Residency Programs}

\author{
Getúlio Rodrigues de Oliveira Filho, TSA $^{1}$
}

\begin{abstract}
RESUMO
Oliveira Filho GR - Bases Teóricas para a Implementação do Aprendizado Orientado por Problemas na Residência Médica em Anestesiologia
\end{abstract}

\begin{abstract}
Justificativa e Objetivos - O Aprendizado Orientado por Problemas $(A O P)$ é um método de ensino cujo objetivo primário é a acumulação de conceitos médicos no contexto de problemas clínicos, que tem sido largamente empregado na graduação médica desde os anos sessenta. O AOP é baseado na teoria do processamento de informação, segundo a qual, a aquisição de novos conhecimentos é facilitada pela ativação de conhecimentos preexistentes sobre o assunto, pela similaridade entre os contextos de aprendizado e aplicação do conhecimento e pela elaboração da informação. Outras teorias têm sido utilizadas para justificar o uso do método AOP no ensino de estudantes de Medicina e em outras áreas. O método AOP utiliza os conceitos aplicados ao aprendizado de adultos, os quais se aplicam aos episódios de aprendizado de médicos.

Conteúdo - Este artigo descreve o método AOP, suas bases teóricas e psicológicas, o papel de instrutores e estudantes no processo e sugestões quanto à sua implementação.

Conclusões - Comparado ao ensino tradicional, o AOP tem como principais vantagens a maior satisfação dos estudantes e instrutores, a maior diversificação das fontes de consulta e busca de conhecimento e o maior tempo despendido no estudo individual. Uma vez que o método não apresenta desvantagens, comparado ao método tradicional, ele pode ser considerado uma alternativa válida para o ensino da Anestesiologia na Residência Médica.
\end{abstract}

UNITERMOS: ANESTESIOLOGIA: aprendizado, ensino

\author{
SUMMARY \\ Oliveira Filho GR - Theoretical Basis for the Implementation of \\ Problem-Oriented Learning in Anesthesiology Residency Pro- \\ grams
}

Background and Objectives - Problem-oriented learning $(P O L)$ is a teaching method the primary objective of which is the accumulation of medical concepts in the context of clinical problems, and which has been widely used in medical graduation since the 60s. POL is based on the information processing theory where acquisition of new knowledge is made easier by activating preexisting knowledge about the subject, by the similarity between learning contexts and knowledge application and by information improvement. Other theories have been evoked to justify POL method in Medical teaching and in other areas. The POL method uses adult teaching concepts applied to medical learning episodes.

Contents - This article describes the POL method, its theoretical and psychological basis, the role of professors and students in the process and suggestions for its implementation.

Conclusions - As compared to traditional methods, POL has as major advantages a higher level of students and professors satisfaction, a wider source of queries and search for knowledge and more time spent with individual learning. Since the method has no disadvantages as compared to traditional methods, it could be considered a valid alternative for teaching Anesthesiology in Medical Residency Programs.

KEY WORDS: ANESTHESIOLOGY: learning, teaching

\section{INTRODUÇÃO}

CET/SBA Integrado de Anestesiologia da Secretaria de Estado da Saúde do Estado de Santa Catarina adotou o Aprendizado Orientado por Problemas (AOP), como

\footnotetext{
* Recebido do (Received from) CET/SBA Integrado de Anestesiologia da SES-SC - Florianópolis, SC

1. Co-responsável pelo CET/SBA Integrado de Anestesiologia da SES-SC - Florianópolis, SC
}

Apresentado (Submitted) em 18 de março de 2002 Aceito (Accepted) para publicação em 04 de junho de 2002

Correspondência para (Mail to):

Dr. Getúlio Rodrigues de Oliveira Filho

Rua Luiz Delfino 111/902

88015-360 - Florianópolis, SC

E-mail: grof@th.com.br

(c) Sociedade Brasileira de Anestesiologia, 2003 método de ensino, em 1999, já tendo formado duas turmas de anestesiologistas.

A razão primordial para a adoção do AOP foi a necessidade do CET de possuir uma filosofia de ensino e um método que incentivassem os instrutores a buscar o aprimoramento didático. Por outro lado, o AOP atraiu a atenção dos instrutores do CET por atender aos princípios de aprendizado de adultos, centrando a responsabilidade da formação da base de conhecimentos no médico residente e estimulando o aprendizado auto-dirigido. A forma de condução do aprendizado é mais descontraída e envolve a participação ativa dos médicos residentes. A Anestesiologia é uma especialidade, na qual resolver problemas faz parte do trabalho diário e a idéia de utilizar estes problemas como ponto de partida para o aprendizado das bases teóricas da especialidade, ou seja, o programa teórico exigido pela Sociedade Brasileira de Anestesiologia, pareceu muito atrativa e desafiadora. 
Num primeiro passo, foram providenciadas salas de aula mais confortáveis, foi instalada uma biblioteca de Anestesiologia e computadores nas dependências do Centro Cirúrgico do hospital-sede, com acesso à Internet, para facilitar a recuperação de material de estudo. ABiblioteca Virtual da Sociedade Brasileira de Anestesiologia muito tem contribuído para a satisfação dos instrutores e residentes com o método, por permitir o acesso imediato à informação de alta qualidade, o mesmo se podendo dizer da Biblioteca da Sede da SBA, dos artigos de revisão da Revista Brasileira de Anestesiologia e, sobretudo, dos registros eletrônicos destes artigos, distribuídos pela SBA.

Paralelamente, foi realizado um treinamento intensivo de instrutores, cobrindo conhecimentos sobre o método em si e noções de psicologia cognitiva, grupal, administração de tempo, e outros tópicos considerados de importância.

Durante este período, as avaliações periódicas e informais mostraram que o método foi rapidamente assimilado pelos instrutores e que os médicos residentes expressaram mais satisfação do que descontentamento com o processo de aprendizado. Houve melhora do desempenho dos médicos residentes nas avaliações anuais promovidas pela Sociedade Brasileira de Anestesiologia e a pontuação do CET meIhorou significativamente, em relação aos anos anteriores.

É claro que o pouco tempo de funcionamento do método no CET não nos permite atribuir ao AOP estes resultados, ainda porque muitos aspectos precisam ser melhorados, especialmente o desempenho dos instrutores.

Este artigo reúne noções teóricas sobre o AOP que se mostraram úteis para a implantação e condução do método. $O$ objetivo deste artigo é informar instrutores e médicos residentes que queiram refletir sobre o processo de aprendizado e que talvez estejam dispostos encarar o desafio da adoção de uma nova filosofia de ensino.

\section{O APRENDIZADO ORIENTADO POR PROBLEMAS}

O aprendizado orientado por problemas (AOP) é um método de ensino cujo objetivo primário é a acumulação de conceitos médicos no contexto de problemas clínicos ${ }^{1,2}$, ou, em termos de psicologia cognitiva, a construção de redes semânticas de conhecimento ajustadas a situações práticas ${ }^{3,4}$. Assim, o método visa (1) implementar o raciocínio clínico e as capacidades em resolver problemas, (2) melhorar a aquisição, a retenção e o uso do conhecimento, (3) aproximar ciências básicas e clínicas, (4) estimular o aprendizado auto-dirigido, (5) estimular o interesse no assunto (motivação), e (6) estimular estratégias mais eficazes de aprendizado ${ }^{3,5}$.

No AOP, o ensino é centrado no aluno e auto-dirigido, ou seja, a responsabilidade pelo aprendizado é do aluno, cuja tarefa é a de construir uma base sólida de conhecimentos através do estudo individual, com o objetivo de reduzir ao máximo a discrepância entre o conteúdo programático e o conhecimento preexistente. O AOP opera através de sessões periódicas de discussão em pequenos grupos destinadas, primariamente, à ativação de conhecimentos prévios dos membros do grupo, à definição dos objetivos de aprendizado e de estratégias de aquisição de conhecimento e à subseqüente elaboração do conhecimento recém adquirido. Ao contrário do método de ensino tradicional, centrado no professor, que é responsável pelo ensino e propõe problemas após a exposição do conteúdo programático, o AOP utiliza problemas encontrados na prática clínica como ponto de partida para a aquisição de novos conhecimentos que expliquem sua etiologia e fisiopatologia e permitam sua solução. Assim, o conhecimento adquirido no AOP transcende a esfera departamental e tem caráter interdisciplinar ${ }^{1}$.

No AOP, o instrutor tem o papel de facilitador do aprendizado, orientando os estudantes durante seu processo individual de busca de conhecimentos e assegurando o bom funcionamento do grupo.

\section{TEORIAS DO APRENDIZADO COGNITIVO}

Os resultados de pesquisas sobre o aprendizado cognitivo podem ser resumidos em cinco teoremas fundamentais:

1.0 conhecimento prévio sobre determinado assunto é o principal determinante da natureza e da quantidade de nova informação que pode ser processada;

2.O conhecimento é mais facilmente recuperado quando a situação em que vai ser aplicado é semelhante àquela em que foi aprendido;

3.O conhecimento é estruturado. A maneira como é estruturado na memória controla sua acessibilidade. O conhecimento consiste de proposições (afirmações que contêm dois conceitos e sua relação) estruturadas em redes semânticas, que incluem relações de causa, tempo, condição, atributos ou localização e qualificadores de negação, de identidade ou de relação entre classes;

4.O armazenamento de nova informação na memória e sua subseqüente recuperação podem ser melhorados pela elaboração durante o aprendizado. A elaboração dá-se pela discussão, ensino, anotações etc;

5.A motivação para o aprendizado, também chamada curiosidade epistemológica, prolonga o tempo de estudo e melhora o resultado do processo cognitivo ${ }^{3}$.

Pelo menos cinco etapas básicas foram identificadas no processo de aprendizado. Em primeiro lugar, o aluno deve reconhecer áreas de deficiência de conhecimento e estar suficientemente motivado para remediá-la. Segundo, o aluno deve ser capaz de reconhecer situações que desencadeiem o episódio de aprendizado. Em terceiro lugar, o aluno deve definir a abrangência de seu estudo, pela geração de questões específicas. A quarta etapa consiste na busca de informação e a quinta etapa consiste na avaliação crítica da informação obtida, quanto à sua relevância e validade para responder às questões feitas durante a terceira etapa. Uma etapa adicional, cujo objetivo é reforçar o conhecimento adquirido nas etapas anteriores, consiste na aplicação deste conhecimento em situações práticas reais ${ }^{6}$. 
A motivação para o aprendizado reside na gratificação, que pode ser externa, como a obtenção de bons resultados em provas de conhecimento, ou interna, como a satisfação pessoal e o senso de dever cumprido. Assim, para criar uma estrutura no processo de aprendizado e para garantir a aquisição de capacidades nos domínios do raciocínio clínico, método científico e avaliação, o aluno deve ser capaz de (1) definir, dirigir e avaliar seu aprendizado, (2) formular questões lógicas e pertinentes que definam a abrangência e a especificidade do episódio de aprendizado e (3) apreciar a aplicabilidade do conhecimento adquirido em situações práticas reais ${ }^{6}$.

Para atingir estas metas, o planejamento curricular deve compreender: (1) a especificação dos objetivos do aprendizado, (2) a forma de proporcionar o aprendizado e (3) a forma de avaliação do aprendizado. Os objetivos do aprendizado não devem ser excessivamente detalhados, a fim de não imporem uma estrutura rígida que remova dúvidas a respeito do que deve ser aprendido. A forma de proporcionar o aprendizado deve estimular a independência progressiva dos alunos em relação ao professor, permitindo liberdade progressiva para que o aluno defina a sua própria abrangência e estratégia de aprendizado. As avaliações formais freqüentes tendem a inibir o aprendizado independente, fazendo com que o estudante se atenha apenas ao conteúdo programático necessário para passar nos testes de conhecimentos. A avaliação informal, por outrolado, permite que o aluno se defronte com suas deficiências de conhecimento e capacidades e seja incentivado a saná-las. Este processo pode ser complementado por testes que requeiram a demonstração da capacidade em resolver problemas e em aplicar o conhecimento a novas situações ${ }^{6}$.

Adultos são motivados a aprender o que é percebido como relevante e pode ser imediatamente posto em prática. Assim, são problemas de ordem prática que desencadeiam os episódios de aprendizado. O processo de aprendizado de adultos é baseado em experiências prévias, é focado em problemas, permite que tomem a iniciativa do aprendizado e compreende ciclos de ação e de reflexão ${ }^{7}$.

$\mathrm{O}$ aprendizado auto-dirigido ocorre quando o estudante toma a iniciativa e a responsabilidade pelo seu próprio aprendizado, diagnosticando suas necessidades de aprendizado, formulando suas metas, identificando fontes de aquisição de conhecimento, implementando atividades e estratégias apropriadas e avaliando os resultados. Em muitos aspectos, os elementos-chave do aprendizado auto-dirigido concordam com os princípios de aprendizado de adultos. $O$ aprendizado auto-dirigido é um processo ativo que estimula a busca do conhecimento profundo, em contraste com o conhecimento superficial, que apenas encoraja os estudantes a reproduzir o que foi aprendido ${ }^{7,8}$.

Médicos aprendem estimulados por problemas específicos (como os de pacientes, por exemplo) ou gerais (necessidade de atualização ou de domínio de novas técnicas, por exemplo). Cada tipo de problema está associado a formas particulares de aquisição de conhecimentos: o aprendizado semi-estruturado, que é utilizado para a solução de proble- mas específicos e compreende consulta a fontes disponíveis de literatura e especialistas, e o aprendizado formal, que consiste em projetos de aprendizado programados, como cursos e congressos. O aprendizado desencadeado por problemas específicos resulta em adições incrementais ao conhecimento e capacidade prévia do médico, enquanto o aprendizado desencadeado por problemas gerais tende a introduzir modificações da prática clínica. Cada episódio de aprendizado se desenvolve em quatro estágios: estágio 0 procura por problemas, em que o médico examina seu ambiente buscando problemas capazes de precipitar o aprendizado ou por idéias e propostas que possam ser úteis no futuro, embora sem aplicação prática atual; estágio 1 - avaliação do problema e decisão sobre a utilidade de aprender sobre ele; estágio 2 - aprendizado de conhecimentos e capacidades necessárias para a solução do problema; estágio 3 - ganho de experiência, pela aplicação do conhecimento. O aprendizado pode ser encerrado no estágio 1, quando o médico acha que não existe solução para o problema, ou prosseguir pelos estágios 2 e 3 , onde pode ser encerrado, mesmo sem haver esgotado as fontes de consulta, se a solução para o problema for obtida. As fontes de referência para a solução de problemas específicos possuem alguns atributos: (1) acessibilidade, fontes de conhecimento facilmente localizáveis no seu ambiente de trabalho, (2) aplicabilidade, a informação localizada está claramente relacionada com o problema, (3) familiaridade com a fonte de aprendizado. As fontes utilizadas para a obtenção de conhecimentos sobre problemas gerais não estão imediatamente disponíveis, pois necessitam de planejamento prévio, já que incluem a participação em congressos, cursos e outras atividades de educação continuada ${ }^{9-11}$.

Conclui-se, portanto, que o médico busca conhecimento somente após resolver se o objeto do aprendizado constitui-se em um problema para ele ou para sua prática. Daí, depreende-se que aqueles que ensinam médicos devem prover informações que permitam e incentivem o médico a considerar pertinente a aquisição de conhecimento. Fica claro, também que o aprendizado médico é ativo, auto-dirigido e orientado à solução de problemas.

O raciocínio clínico se desenvolve em quatro estágios, de acordo com o grau de exposição a pacientes:

1.Estágio 1 - Desenvolvimento de redes causais elaboradas: em que os estudantes desenvolvem rapidamente redes de causas e conseqüências de doenças em termos de processos fisiopatológicos subjacentes;

2.Estágio 2 - Compilação de redes elaboradas em redes condensadas: através do uso repetido e do contato com pacientes, as redes existentes são compiladas em modelos causais simplificados que explicam sinais e sintomas que compõem os rótulos diagnósticos e contêm apenas os conceitos mais relevantes da rede fisiopatológica original;

3.Estágio 3 - Emergência de scripts de doenças: simultaneamente à compilação, ocorre a transição do modelo causal de organização do conhecimento para a 
estruturação em listas de rótulos diagnósticos ou modelos mentais simplificados que são suficientes para explicar o fenômeno observado em termos de diagnóstico e tratamento. Estes modelos são chamados scripts de doenças. Um script é formado pelos seguintes componentes: (1) condições favorecedoras, que incluem fatores predisponentes e limitantes à ocorrência da doença, (2) falha, que contém os defeitos de funcionamento de órgãos ou sistemas causados pela doença, e (3) conseqüências, que representam as manifestações clínicas. Num script de doença, não estão geralmente presentes princípios fisiopatológicos, o que permite concluir que, ao se lidar com casos rotineiros, o processo de solução de problemas de pacientes limita-se à busca, seleção e verificação de scripts constantes das estruturas de memória do médico e aplicáveis ao problema do paciente. Os scripts variam de médico para médico e guardam pouca relação com casos prototípicos apresentados em livros-texto, já que são criados a partir da experiência clínica do médico;

4.Estágio 4 - Criação de scripts exemplares - pacientes anteriores, suas características e situações em que foram atendidos são armazenados na memória como exemplos que representam os diversos padrões de apresentação de determinada doença. Nesta fase, o médico manuseia os problemas pelo reconhecimento de padrões de doença ${ }^{12}$.

As primeiras hipóteses sobre o processo de solução de problemas eram de que: (1) os problemas médicos representavam um conjunto relativamente homogêneo de situações úteis ao desenvolvimento de capacidades para a solução de problemas, (2) o processo de solução de problemas era único e aplicável a todos os problemas médicos, e (3) o processo era uma variante do método científico hipotético-dedutivo. No entanto, pesquisas subseqüentes revelaram que: (1) o sucesso da solução de problemas depende mais do domínio do conhecimento específico sobre o problema do que da estratégia utilizada para sua solução, (2) uma das estratégias utilizadas por especialistas para a solução de problemas é a do reconhecimento de padrões de doença, a qual não envolve a utilização de ciências básicas, e (3) especialistas utilizam tanto o raciocínio anterógrado (dos dados clínicos para as hipóteses diagnósticas), quanto o retrógrado (das hipóteses diagnósticas para os dados clínicos), dependendo da complexidade do problema. Assim, problemas rotineiros podem ser resolvidos com base no reconhecimento de padrões ou raciocínio anterógrado, enquanto problemas complexos elicitam o raciocínio retrógrado, característico do método hipotético-dedutivo. O raciocínio anterógrado de especialistas baseia-se na existência de esquemas específicos para cada problema dentro de seu domínio de conhecimento. Neste contexto, um esquema é uma categorização mental do conhecimento que inclui um modo particular e organizado de compreender e responder a uma situação complexa. Autilização de esquemas explanatórios durante a fase de aprendizado permite que estes mesmos esquemas sejam utiliza- dos posteriormente para recuperar informações da memória durante a fase de solução de problemas diagnósticos, ou seja, os esquemas, se utilizados tanto durante o aprendizado quanto durante a solução de problemas reais, têm a vantagem de combinar a criação de uma estrutura mental de conhecimento e uma estratégia de busca e recuperação, em um mesmo processo. Uma vez que a organização do conhecimento em esquemas baseia-se na identificação e integração de conceitos básicos em mecanismos fisiopatológicos que possam explicar os dados clínicos presentes no problema, pode-se concluir que sua utilização durante o aprendizado cognitivo de residentes favorece a integração entre ciências básicas e clínicas, além de permitir a estruturação do conhecimento e de facilitar a sua recuperação ${ }^{13}$.

\section{BASES TEÓRICAS DO APRENDIZADO ORIENTADO POR PROBLEMAS}

O AOP tem como principal base teórica a teoria de processamento de informação, segundo a qual três princípios orientam a aquisição de novas informações: (1) ativação de conhecimentos prévios sobre o assunto, (2) especificidade de codificação (a recuperação de informação no futuro é facilitada quando exemplos estão codificados juntamente com a informação, ou seja, quanto mais próxima a semelhança entre uma situação real e a situação de aprendizado, mais fácil a recuperação de informação), e (3) elaboração do conhecimento (através de resposta a perguntas, ensino de colegas ou verbalização do conhecimento), que cria redundância na estrutura da memória ${ }^{1}$.

Outras teorias têm sido invocadas para justificar o uso do AOP $^{14}$ :

Ateoria do aprendizado cooperativo sugere que o aprendizado individual é mais eficaz quando o indivíduo percebe que só atingirá suas metas de aprendizado se os demais membros de seu grupo também o fizerem. Esta postura contrasta com o postura competitiva. Grupos engajados no aprendizado cooperativo explicam mais e corrigem os erros uns dos outros do que grupos competitivos, propiciando maior elaboração do conhecimento.

A teoria da autodeterminação reconhece dois tipos de motivação: a controlada, imposta por demandas externas e orientada para premiações e punições; e a autônoma, orientada para o que o indivíduo percebe como importante ou interessante. O indivíduo estuda simplesmente porque sente-se gratificado em fazê-lo. A motivação autônoma leva a melhor entendimento conceptual, melhor desempenho acadêmico, sentimentos mais fortes de competência, aumento da criatividade, preferência por desafios, em vez do sucesso fácil, maior persistência e maior ajuste psicológico.

Aaquisição de conhecimentos e seu armazenamento na memória baseiam-se em três princípios fundamentais da psicologia cognitiva: (1) a ativação de conhecimentos prévios facilita o processamento de novas informações; por outro lado, a recuperação do conhecimento no futuro é facilitada (2) pela elaboração do conhecimentos através de discussão, notas, respostas a perguntas, ensino de colegas ou sua utilização 
para a compreensão de um problema e (3) pela similaridade entre os contextos em que o conhecimento é adquirido e aplicado. Um aspecto importante da psicologia cognitiva aplicada ao AOP refere-se à capacidade de transferência de conhecimento entre problemas que requeiram o mesmo conhecimento, mas sejam apresentados de maneiras distintas. Para que tal ocorra, duas condições precisam ser satisfeitas: (1) é necessário que os passos que levam à identificação e análise do problema sejam rigorosamente seguidos pelos estudantes ao invés de serem apresentados pelo instrutore (2) retroalimentação corretiva deve ser dada pelo instrutor durante as tentativas de solução dos problemas, evitando-se a formação de falsos conceitos ${ }^{15}$.

\section{O PROCESSO DE AOP}

Classicamente, as sessões de AOP obedecem a uma rotina, resumida em sete passos, a partir de um problema não estruturado e aberto, ou seja, somente as palavras-chave são apresentadas, e nenhuma ou pouca informação é fornecida, a fim de permitir a avaliação multi-dimensional do problema: (1) clarificação dos termos e conceitos que não sejam prontamente compreensíveis, (2) definição do problema, (3) análise do problema, utilizando conhecimento prévio, (4) elaboração de um inventário sistemático, a partir das explicações obtidas no passo 3, (5) formulação dos objetivos do aprendizado e das estratégias para obtenção de informações, (6) coleta de informações, individualmente e (7) síntese e teste da informação nova adquirida ${ }^{1}$.

A aplicação de novos conhecimentos, especialmente a emergência do conceito de esquemas ou algorritmos utilizados por especialistas para a solução de problemas, gerou uma nova maneira de conduzir as sessões de AOP, que introduz a construção de esquemas ou algorritmos como o meio para busca de conhecimento e solução do problema. O processo pode ser resumido em sete etapas ${ }^{16}$ :

1.Identificação do problema;

2.Desenvolvimento de uma abordagem lógica ao processo diagnóstico e identificação dos assuntos-alvo do aprendizado, aplicáveis à construção de um algorritmo destinado à solução do problema;

3. Coleta individual de informações;

4.Derivação de um algorritmo para a solução do problema, utilizando as informações coletadas;

5.Aplicação do algorritmo para a elucidação diagnóstica, incluindo dados de história, exame físico e complementares;

6.Discussão dos dados relevantes, ativação de conceitos prévios, elaboração das interpretações individuais dos dados, reorganização destes dados e conclusão diagnóstica;

7.Apresentação e solução de outros casos, representativos do mesmo problema.

\section{FORMULAÇÃO DE PROBLEMAS}

Os problemas devem ser cuidadosamente elaborados, com a finalidade de obedecer às finalidades do aprendizado, devendo possuir as seguintes características: (1) consistir de uma descrição neutra de uma situação (intercorrência ou doença) que precise de explicação de mecanismos, processos subjacentes ou princípios, relacionados com o conteúdo programático; (2) permitir discussão, evitando-se as perguntas de natureza direta; (3) retratar situações que possam ser encontradas na prática; (4) ser compatíveis com o nível de conhecimento dos residentes; (5) incluir intercorrências mais prevalecentes; (6) representar situações críticas ou urgentes; (7) devem estar associados com alta morbidade ou mortalidade; (8) incluir problemas reconhecidamente mal manuseados ou pouco conhecidos na comunidade ${ }^{1}$.

Outros autores ${ }^{17}$ utilizaram a técnica Delphi, na qual especialistas julgam, definem e priorizam características, através de consenso, para definir as características mais importantes de um problema. Segundo estes autores, os problemas devem (1) estimular o pensamento, análise e raciocínio, (2) assegurar o aprendizado auto-dirigido, (3) incentivar o uso de conhecimentos prévios, (4) serem propostos em contextos reais, (5) levar à descoberta dos objetivos do aprendizado, (6) despertar a curiosidade, (7) referir-se a temas relevantes à especialidade, (8) assegurar amplitude contextual, através de detalhes que identifiquem a situação e (9) favorecer o uso e aquisição de vocabulário científico.

\section{O PAPEL DOS INSTRUTORES, DOS ESTUDANTES E DA INSTITUIÇÃO}

No AOP, os instrutores assumem o papel de facilitadores do aprendizado. Assim, a primeira mudança deve ocorrer no corpo de instrutores, que deverão adquirir conhecimentos que os habilitem a exercer suas funções de tutores dos grupos de estudantes. A principal dificuldade encontrada pelos instrutores, em relação ao tutoramento de grupos, reside em assumir o papel de facilitador, ao invés do de professor ${ }^{18}$. Tutores que são especialistas no assunto tendem a ser excessivamente diretivos e a explicar demais, impedindo que os estudantes desenvolvam interações produtivas durante as sessões de AOP $^{19,20}$. O desconhecimento de processos grupais é outra causa de insucesso de instrutores, uma vez que, ao permitir que o grupo interaja, mecanismos grupais e crises aparecem facilmente. O desconhecimento destes mecanismos e seu manuseio faz com que os instrutores assumam posturas diretivas e professorais, prejudicando o processo de aprendizado do grupo ${ }^{21}$.

Os estudantes têm manifestado preferência por tutores que dominem o assunto, que façam questões pertinentes, que orientem o raciocínio, que se entrosem com o grupo, que dêem feed-back corretivo e que não tolham a espontaneidade dos estudantes. Esta última característica é essencialmente obtida pela postura receptiva do tutor em relação às opiniões e assertivas dos estudantes, evitando a crítica intimidatória ${ }^{22,23}$. 
A implantação de um programa baseado no AOP precisa contar com o comprometimento dos instrutores, no campo ideológico. Uma vez conseguido isto, parte-se para a formação teórica e prática dos instrutores interessados em participar do programa. Esta formação, normalmente realizada utilizando o próprio método, compreende tópicos como as bases pedagógicas e psicológicas do AOP, a construção de problemas, mecanismos grupais, formas de avaliação, liderança situacional, administração do tempo, diagnóstico e manuseio de grupos disfuncionais, entre outros, de acordo com as características das instituições e de seu corpo de instrutores. É importante que os instrutores definam, à medida que são expostos ao treinamento, conteúdos adicionais que supram suas próprias necessidades de aprendizado. Estes treinamentos têm sido avaliados muito positivamente pelos instrutores participantes quanto à sua aplicabilidade ao processo tutorial ${ }^{16,24-27}$.

O estilo de liderança a ser desempenhado pelo tutor depende, fundamentalmente, da maturidade do grupo, que se manifesta pelo grau de segurança de seus membros, por sua capacidade e motivação para desempenhar as tarefas e pela quantidade e tipo de feed-back de que necessitam. Foram identificados quatro estilos de liderança, aplicáveis a diferentes tipos de grupos:

1.Diretiva, utilizada para grupos imaturos, com pouca capacidade e motivação para executar as tarefas propostas, enfatiza a obtenção dos resultados, com pouca ênfase na esfera social do grupo;

2.Educadora, utilizada para grupos com maturidade baixa a moderada, caracterizados pela disposição em aprender, apesar da falta de capacidades, enfatiza o entendimento da tarefa;

3. Motivadora, utilizada para grupos com maturidade moderada para alta, caracterizados pela falta de disposição em realizar as tarefas, apesar da existente competência, enfatiza a participação dos elementos do grupo no processo de tomada de decisões, como estratégia motivacional;

4.Delegadora, utilizada para grupos altamente maduros, caracterizados pela competência e disposição em realizar as tarefas, enfatiza a delegação de responsabilidades aos membros do grupo e caracteriza-se pela pouca ação facilitadora do líder.

Cada estilo de liderança utiliza diferentes tipos de poder para obter resultados do grupo. Assim, o estilo diretivo utiliza o poder coercitivo, baseado em punições; o estilo educador, o poder de recompensas pelo bom desempenho; o estilo motivador utiliza o poder referencial, baseado na identificação dos elementos do grupo com o líder. No estilo delegador, os membros percebem o líder como o elemento mais competente do grupo, sendo o respeito a esta excelência o elemento de poder que norteia as relações com o líder. É importante assinalar que, em diferentes situações, quando confrontados com diferentes tipos de problemas, pressionados por fatores externos ou conflitos internos, os membros do grupo adotarão posturas mais ou menos maduras, o que exige que o líder esteja treinado para reconhecer o grau de maturidade do grupo em cada momento, para adotar o estilo de liderança que seja mais apropriado à situação, a fim de que o desempenho do grupo não seja comprometido ${ }^{28}$.

A exposição dos estudantes ao AOP deve ser precedida de instruções quanto ao método e suas justificativas pedagógicas. Os estudantes devem ser instruídos sobre a dinâmica do processo de aprendizado, com noções sobre os passos a serem seguidos, formas de avaliação, fontes de obtenção de informações, incluindo membros do corpo de instrutores e suas áreas particulares de interesse, a fim de que possam ser utilizados como fontes de conhecimento ${ }^{29}$.

Cabe à instituição garantir conforto, ambiente seguro e fontes de aprendizado, incluindo biblioteca, acesso à Internet e material audiovisual, que serão utilizadas pelos estudantes para o aprendizado. É conveniente lembrar que as sessões de AOP possuem duração de duas a três horas, de tal forma que cadeiras confortáveis, iluminação adequada e ar condicionado são essenciais para evitar a fadiga física que ocorre em situação de desconforto, má iluminação e inadequação térmica e que contribui para o menor rendimento do aprendizado.

Um aspecto fundamental da implantação o AOP é a discussão do novo currículo, cujas características atendam às necessidades do método. Novas taxonomias de conteúdos curriculares têm sido desenvolvidas, através de ampla discussão do corpo docente da instituição, formação de comitês curriculares centrais ou delegação de responsabilidades aos departamentos da instituição ${ }^{30-33}$.

Nem todos os grupos funcionam produtivamente. A falta de produtividade caracteriza o grupo disfuncional. A manifestação mais freqüente de disfunção grupal é o comportamento ritual, que ocorre quando os estudantes mantêm a falsa aparência de envolvimento ativo na solução dos problemas propostos, durante as sessão de AOP. Este comportamento externa-se (1) pela ausência de conexão entre as idéias novas e a base de conhecimentos prévios, (2) pela geração de objetivos de aprendizado não claramente definidos ou sem relação específica com o problema em questão e (3) pelo estudo individual deficiente. Como conseqüência deste comportamento, tanto a ativação de conhecimentos prévios quanto a elaboração do conhecimento não ocorrem, impedindo o progresso do processo de aprendizado. Tutores não treinados em reconhecer e lidar com este tipo de comportamento tendem a sentirem-se frustrados e tentam remediar a falta de progresso do grupo tomando o papel de professor ou delegando-o a um dos membros do grupo, ou seja, adotando o método da educação tradicional. Esta atitude rompe a base filosófica do AOP e reduz a auto-estima do grupo. As estratégias propostas para a solução deste tipo de disfunção sugerem que o tutor: (1) proponha problemas mais complexos, que exijam maior interação entre os membros do grupo, (2) proponha questões que ativem a recuperação de conhecimentos prévios dos membros do grupo e sua aplicação para a solução do problema em questão, (3) formule, com os estudantes, os objetivos de aprendizado de forma clara e especí- 
fica, (4) estimule a expressão de todos os membros do grupo e o feed-backaos colegas e (5) estimule os estudantes a aplicar os novos conhecimentos a outros problemas ${ }^{34}$.

Alguns membros do grupo, podem adotar o papel de parasi$\underline{\text { tas, }}$, ao não participar ativamente do trabalho, deixando que os outros membros do grupo o façam por ele. Estes, por sua vez, ao se darem conta deste tipo de comportamento, passarão a contribuir cada vez menos com as atividades do grupo, caracterizando o comportamento de retirada. Assim, a coesão grupal se rompe e a produtividade cai. Uma outra forma de comportamento disfuncional se caracteriza pela aceitação excessivamente fácil de explicações pelos membros do grupo ou pela explicação inadequada dos tópicos constantes dos objetivos do aprendizado ${ }^{35}$. Nestas situações, cabe ao tutor e ao grupo identificar e discutir esses comportamentos, suas repercussões sobre o processo de aprendizado e alternativas para solucioná-los.

Os tutores também podem contribuir para as disfunções grupais ao adotarem posturas autocráticas ou excessivamente diretivas durante as sessões de AOP. Além disto, no outro extremo, tutores que não se mostram comprometidos com o trabalho do grupo (líderes laissez-faire ou não-líderes) permitem a perpetuação de falsos conceitos, o que compromete o processo de aprendizado e reduz a produtividade grupal ${ }^{28}$. Um estudo sobre as percepções de estudantes sobre o tutoramento de AOP revelou que os estudantes esperam que o tutor seja um facilitador hábil que os guie durante o aprendizado e ao mesmo tempo mantenha um clima grupal positivo. Os estudantes não querem que os tutores ensinem o conteúdo programático, já que o processo de aprendizado foi percebido como tarefa deles ${ }^{22,36,37}$.

Afalta de feed-back compromete o processo de aprendizado por permitir o aprendizado de conceitos falsos e estratégias erradas de solução de problemas. Afalta de feed-back corretivo contribui para a falha de transferência entre problemas ${ }^{15}$. O feed-back entre os membros do grupo deve ser incentivado, bem como o feed-back individual dado ao estudante pelo tutor. O feed-back deve ter conotação corretiva e estimulante e deve ser dado ao grupo durante todas as sessões de AOP (feed-back imediato) ${ }^{38,39}$.

\section{O AOP É MELHOR QUE O MÉTODO TRADICIONAL?}

Não existem dados referentes aos efeitos do AOP sobre o aprendizado na residência médica em Anestesiologia. Cinco revisões extensas, comparando o AOP com o ensino tradicional durante o curso de graduação em Medicina, foram realizadas entre 1993 e 2000 , utilizando métodos descritivos ou metanalíticos. Diversos aspectos foram estudados e os resultados são resumidos a seguir:

Albanese ${ }^{40}$, utilizando a abordagem metanalítica e revisão sistemática dos estudos existentes até a data, concluiu que alunos de programas de graduação que utilizavam o método apresentavam desempenho pior em exames de ciências básicas e desempenho igual ou melhor em exames de ciências clínicas do que alunos de programas tradicionais. Utilizavam predominantemente raciocínio retrógrado (dedução a partir de soluções hipotéticas para explicar os dados observados), possuíam estratégias de ensino mais versáteis, mais horas de estudo, maior freqüência a biblioteca. Quanto à avaliação dos estudantes e preceptores, os autores encontraram percepção mais positiva do ambiente de aprendizado, maior satisfação dos estudantes e percepção de preparo pelos graduados, embora se achando deficitários em termos de ciências básicas. Quanto aos percentuais de obtenção de vagas em primeiras opções de residência médica, estudantes de AOP apresentaram percentuais similares aos do método tradicional. Embora despendendo mais tempo na condução de sessões de AOP, os instrutores tenderam a achar que os benefícios do método são maiores e sentem-se satisfeitos. Os autores apontam ainda algumas restrições e tópicos não resolvidos, como a possibilidade de haver maior custo, quando as turmas são maiores que 40 alunos, a menor velocidade de aquisição de conhecimentos, a possibilidade de não desenvolver adequadamente conhecimentos em ciências básicas e a possibilidade de criar profissionais incapazes de trabaIhar isoladamente.

Vernon ${ }^{41}$, utilizando abordagem metanalítica, concluiu que (1) o resultado das avaliações dos programas favorecem o AOP, (2) não houve diferença entre os métodos quanto às notas obtidas em exames nacionais, (3) alunos do AOP colocam mais ênfase no entendimento dos processos subjacentes aos problemas, enquanto alunos de métodos tradicionais tendem a preocupar-se mais com a reprodução dos conhecimentos. Alunos de AOP tendem a ser mais criativos quanto às fontes de obtenção de conhecimentos e apresentam meIhor desempenho nas áreas clínicas e pior nas áreas básicas do que estudantes de métodos tradicionais.

Berkson ${ }^{42}$, em uma revisão sistemática, concluiu que o AOP não é distinguível do ensino tradicional, podendo ser estressante para os alunos e professores e excessivamente caro para as instituições de ensino.

Thomas ${ }^{43}$, em outra revisão sistemática, concluiu que, no AOP, os estudantes não apresentaram maior motivação para o estudo do que estudantes do método tradicional, que houve predominância de raciocínio retrógrado e que não houve diferenças entre os métodos quanto à capacidade de estruturação do conhecimento no contexto clínico. Entretanto, no AOP, os estudantes apresentaram maior diversificação das fontes de aquisição de conhecimentos.

Colliver ${ }^{44}$, utilizando abordagem metanalítica, concluiu que os efeitos apresentados por metanálises anteriores e por estudos prospectivos são pequenos e estatisticamente insignificantes. Concluiu também, que o AOP fornece uma abordagem mais desafiadora, motivadora e agradável à educação médica, cuja superioridade em relação ao ensino tradicional ainda não foi comprovada. Em sua metanálise, o autor ainda sugere que não existe uma base teórica firme que justifique o AOP. Neste estudo, somente foram considerados significativos, efeitos de tamanho superior a 0,8 desvio padrão, um critério muito mais rigoroso que os utilizados em comparações metanalíticas anteriores e a única teoria considerada foi a do aprendizado contextual ${ }^{14}$. 
Três aspectos emergem como as principais vantagens do AOP: a maior satisfação dos estudantes e instrutores com o método, a maior diversificação das fontes de consulta para obtenção de conhecimentos e o maior tempo despendido no estudo individual.

$\mathrm{Na}$ ausência de desvantagens do método de AOP, em relação ao tradicional, estas razões já são suficientes para sua utilização, levando-se em conta que a satisfação dos médicos residentes e dos instrutores é uma dimensão importante da qualidade da residência médica ${ }^{45,46}$.

\section{Theoretical Basis for the Implementation of Problem-Oriented Learning in Anesthesiology Residency Programs}

\author{
Getúlio Rodrigues de Oliveira Filho, TSA, M.D
}

\section{INTRODUCTION}

The Integrated Anesthesiology CET/SBA of the Health Department of the State of Santa Catarina has adopted in 1999 the Problem-Oriented Learning (POL) method and has already graduated 2 groups of anesthesiologists.

The major reason for POL adoption was the need for a teaching philosophy and method encouraging trainers to look for didactic improvement. On the other hand, POL has called the attention of CET trainers for meeting adult teaching principles, centering the responsibility of building a knowledge base on the resident physician and encouraging self-oriented learning. It is a more relaxed teaching method involving the active participation of resident physicians. Anesthesiology is a specialty where solving problems is part of the daily work and the idea of using these problems as the starting point for learning specialty's theoretical basis, that is, the theoretical program required by the Brazilian Society of Anesthesiology, seemed very attractive and challenging.

As a first step, more comfortable classrooms were provided; an Anesthesiology library and computers were installed within the Surgical Center of the parent hospital, with access to the Internet to make easier the retrieval of study material. The Brazilian Society of Anesthesiology's Virtual Library has contributed to the satisfaction of tutors and students with the method for allowing immediate access to high quality information, the same being true for SBA headquarter's library, review articles of the Brazilian Journal of Anesthesiology and, especially electronic records of these articles made available by SBA.

In parallel, tutors were intensively trained on the method itself and on notions of cognitive and group psychology, time management and other important subjects.

During this time, periodic and informal evaluations have shown that tutors had promptly assimilated the method and that resident physicians were more happy than unhappy with the learning process. Annual evaluations by the Brazilian Society of Anesthesiology showed improved performance of resident physicians and CET score has significantly improved as compared to previous years.

Of course, the recentness of the method does not allow us to attribute such results to POL, even because several aspects have to be improved, especially tutors' performance.

This article gathers POL theoretical notions which have been proven useful for the implementation and conduction of the method. This article aimed at informing tutors and resident physicians wishing to think about the learning process and who might be willing to face the challenge of adopting a new teaching philosophy.

\section{PROBLEM-ORIENTED LEARNING}

Problem-oriented learning (POL) is a teaching method aiming primarily at accumulating medical concepts within the context of clinical problems ${ }^{1,2}$ or, in cognitive psychology terms, the building of semantic knowledge networks adjusted to practical situations ${ }^{3,4}$. So, the method aims at (1) implementing clinical reasoning and problem solving skills; (2) improving knowledge acquisition, retention and utilization; (3) bringing basic and clinical science together; (4) encouraging self-oriented learning; (5) encouraging the interest on the subject (motivation); and (6) encouraging more effective learning strategies ${ }^{3,5}$.

With POL, teaching is student-centered and self-oriented, that is, students are responsible for learning and their task is to build a sound knowledge base through individual study, aiming at decreasing to a minimum the discrepancy between program content and preexisting knowledge. POL operates through periodic small group discussion sessions primarily aimed at the activation of previous knowledge of the group, at the definition of learning objectives and knowledge acquisition strategies, and at fine-tuning recently acquired knowledge. As opposed to traditional professor-centered learning, where the professor is in charge of teaching and poses problems after explaining the subject, POL uses problems found in the clinical practice as the starting point for the acquisition of new knowledge explaining their etiology and pathophysiology and allowing for their solution. So, the knowledge acquired via POL goes beyond the departmental sphere and has an interdisciplinary character ${ }^{1}$.

POL tutors act as learning facilitators, orienting students during their search for individual knowledge and assuring the good performance of the group.

\section{COGNITIVE LEARNING THEORIES}

Cognitive learning research results may be summarized in five fundamental theorems:

1.Previous knowledge about a subject is the major determinant of the nature and amount of new information able to be processed; 
2.Knowledge is more easily retrieved when the situation in which it is going to be applied is similar to that in which it was learned;

3.Knowledge is structured. The way it is structured in memory controls its accessibility. Knowledge is made up of propositions (statements with two concepts and their relationships) structured in semantic networks including cause, time, condition, attributes or location relationships, and denial, identity or class relationship qualifiers;

4. Storage of new information in the memory and its subsequent retrieval may be improved by elaboration during learning. Elaboration is achieved through discussions, teaching, personal notes etc.;

5.Learning motivation, also called epistemologic curiosity, prolongs study time and improves cognitive process results ${ }^{3}$.

At least five basic stages were identified in the learning process. First, students have to recognize knowledge-deficient areas and be motivated enough to remedy them. Second, students must be able to identify situations triggering learning episodes. Third, students should define the comprehensiveness of their study by generating specific questions. The fourth stage consists on the search for information and the fifth stage consists on the review of obtained information as to its relevance and validity to answer questions asked during the third stage. An additional stage, the objective of which is to reinforce knowledge acquired during previous stages, is the application of this knowledge to practical and real situations ${ }^{6}$.

Learning motivation resides in rewards, which may be external, such as good results in knowledge tests, or internal, such as personal satisfaction and a sense of having accomplished a duty. So, to create a learning process structure and to assure the acquisition of clinical reasoning, scientific method and evaluation capabilities, students must be able to (1) define, orient and evaluate their learning; (2) formulate logic and pertinent questions defining the comprehensiveness and specificity of the learning episode; and (3) appreciate the applicability of acquired knowledge to practical and real situations ${ }^{6}$. To meet such objectives, the curricular planning should encompass: (1) specification of learning objectives; (2) a way to provide learning; and (3) how learning will be evaluated. Learning objectives should not be excessively detailed not to impose a rigid structure removing questions about what should be learned. Learning should encourage progressive students independence from the professor, allowing a progressive freedom, for the student to define his own learning comprehensiveness and strategy. Frequent formal evaluations tend to inhibit independent learning, leading students to stick only to the program content needed to be approved in knowledge tests. Informal evaluations, on the other hand, allow students to face their knowledge and capability deficiencies and be encouraged to correct them. This process may be complemented by tests requiring the demonstration of capabilities to solve problems and apply the knowledge to novel situations ${ }^{6}$
Adults are motivated to learn what is perceived as relevant and can be put immediately into practice. These are practical problems triggering learning episodes. Adult learning process is based on previous experiences, is problem-centered, allowing them to take learning initiatives and involving action and reflection cycles ${ }^{7}$.

There is self-oriented learning when students take the initiative and responsibility for their own learning, diagnosing their learning needs, formulating their goals, identifying knowledge acquisition sources, implementing adequate activities and strategies and evaluating results. In several aspects, the key-elements of self-oriented learning are in line with adult learning principles. Self-oriented learning is an active process encouraging the search for deep knowledge, in contrast to superficial knowledge, which only encourages students to reproduce what has been learned ${ }^{7,8}$

Physicians learn encouraged by specific (such as patients' problems, for example) or general (need for updates or mastering of new techniques, for example) problems. Each problem is associated to specific ways of knowledge acquisition: semi-structured learning, used to solve specific problems and involving consultation to available literature sources and experts, and formal learning, consisting of programmed learning projects, such as courses and scientific meetings. Learning triggered by specific problems results in incremental additions to previous knowledge and capability, while learning triggered by general problems tends to introduce modifications in the clinical practice. Each learning episode is developed in four stages: stage 0 - problem search, where the physician evaluates his environment looking for problems able to trigger learning, or for ideas and proposals which may be useful in the future, although without current practical application; stage 1 - evaluation of the problem and decision about the usefulness of learning about it; stage 2 - acquisition of knowledge and capabilities needed to solve the problem; stage 3 gain of experience by knowledge application. Learning may be concluded in stage 1 , if physicians believe there is no solution for the problem, or may proceed to stages 2 and 3 , where it may be finished, even before exhausting reference sources, if the solution is found. Reference sources for specific problem solving have some attributes: (1) accessibility, knowledge sources easily located in the working place; (2) applicability; information is clearly related to the problem; (3) familiarity with the learning source. Sources used to obtain information about general problems are not immediately available because they need previous planning, since they include attending scientific meetings, courses and other continued education activities ${ }^{9-11}$.

The conclusion, then, is that physicians look for knowledge only after deciding whether the learning subject is a problem for them or their practice. So, those who teach physicians should provide information allowing and encouraging physicians to consider relevant the acquisition of that knowledge. It is also clear that medical learning is active, self-oriented and geared toward problem solving.

Clinical reasoning is developed in four stages, according to the level of exposure to patients: 
1.Stage 1 - Developing sophisticated causal networks; where students promptly develop disease cause and consequence networks in terms of underlying pathophysiological processes;

2.Stage 2 - Compiling sophisticated networks into condensed networks: through the repeated use and the contact with patients, existing networks are compiled into causal and simplified models explaining signs and symptoms making up the diagnosis and containing only most relevant concepts of the original pathophysiological network;

3.Stage 3 - Disease scripts: simultaneously to compiling there is the transition from the causal knowledge organization model to structuring into diagnostic label lists or simplified mental models sufficient to explain phenomena observed in terms of diagnostic and treatment. These are called disease scripts. A script is made up of the following components: (1) favoring conditions, including disease predisposing and limiting factors; (2) failure, with the defects of organ or systems function caused by the disease; and (3) consequences, representing clinical manifestations. In general, pathophysiological principles are not part of the disease script, which leads to the conclusion that, in dealing with routine cases, patients' problems solving is limited to the search, selection and checking of scripts which are part of physicians memory structures and may be applied to patients' problems. Scripts vary from physician to physician and have a poor relationship with prototypes shown in text books, since they are created as from physicians clinical experience;

4.Stage 4 - Sample scripts - previous patients, their profiles and situation in which they were seen are stored in the memory as examples representing different presentation patterns of a certain disease. In this stage, physicians handle problems by recognizing disease patterns ${ }^{12}$.

The first hypotheses about the problem-solving process were that: (1) medical problems are a relatively homogeneous set of situations useful for the development of problem-solving capabilities; (2) the problem-solving process is one and applicable to all medical problems; and (3) the process is a variant of the hypothetical-deductive scientific method. Subsequent research, however, has revealed that: (1) problem-solving success depends more on the mastering of specific knowledge about the problem than on the strategy used for its solution; (2) a strategy used by problem-solving experts is to recognize disease patterns, which does not involve basic science; and (3) experts use both anterograde reasoning (from clinical data to diagnostic hypothesis) and retrograde reasoning (from diagnostic hypothesis to clinical data), depending on the complexity of the problem.

So, routine problems may be solved based on recognizing patterns, or anterograde reasoning, while complex problems require retrograde reasoning, characteristic of the hypothetic-deductive method. Experts' anterograde reasoning is based on the existence of specific schemes for each problem within their realm of knowledge. In this context, a scheme is a mental categorization of knowledge including a special and organized way to understand and respond to a complex situation. The use of explanatory schemes during the learning stage allows these same schemes to be used in the future to retrieve information from the memory during diagnostic problems solving stage, that is, schemes, if used both during learning and real problems solving, have the advantage of combining the creation of a mental knowledge structure and a search and retrieve strategy in the same process. Since the organization of knowledge into schemes is a function of the identification and integration of basic concepts into pathophysiological mechanisms able to explain clinical data present in the problem, one may conclude that its use during cognitive learning favors the integration between basic and clinical science, in addition to allowing knowledge structuring and helping its retrieval ${ }^{13}$.

\section{THEORETICAL BASES OF PROBLEM ORIENTED LEARNING}

Primary POL theoretical basis is the information processing theory according to which three principles guide the acquisition of new information: (1) activation of previous knowledge about the subject; (2) specificity coding (future information retrieval is made easier when examples are coded with the information, that is, the closer the similarity between a real situation and the learning situation, the easier the information retrieval); and (3) knowledge elaboration (by answering questions, peers teaching or knowledge verbalization), which creates redundancy in the memory structure ${ }^{1}$.

Other theories have been evoked to justify POL ${ }^{14}$ :

Cooperative learning theory suggests that individual learning is more effective when people realize that they will only meet their learning goals if other members of the group meet them. This position is in contrast with the competitive position. Groups engaged in cooperative learning explain and correct each other mistakes more than competitive groups, thus allowing a better knowledge elaboration.

The self-determination theory recognizes two types of motivation: controlled motivation, imposed by external demands and oriented to award or punishment; and autonomous motivation, oriented toward what people perceive as relevant or interesting. People study only because they feel gratified in doing so. Autonomous motivation leads to better conceptual understanding, better academic performance, stronger competence feelings, increased creativity, preference for challenges as compared to easy success, more persistence and better psychological adjustment.

Knowledge acquisition and storage in the memory are based on 3 basic cognitive psychology principles: (1) the activation of previous knowledge helps the processing of new information; on the other hand, future knowledge retrieval is made easier (2) elaboration of knowledge through discussions, notes, questions answering, peer teaching or knowledge use to understand a problem; and (3) similarity among contexts in which knowledge is acquired and applied. An important aspect of cognitive psychology applied to POL is the ability to 
transfer knowledge among problems requiring the same knowledge, but being presented in different ways. For such, two conditions have to be met: (1) steps leading to problem identification and analysis have to be strictly followed by students and not presented by the tutor; and (2) the trainer shall give corrective feedback during problem-solving attempts, thus preventing misconceptions ${ }^{15}$.

\section{POL PROCESS}

Classically, POL sessions follow a routine, summarized in seven steps as from a non-structured and open problem, that is, only keywords are presented and few or no information is given to allow a multidimensional evaluation of the problem: (1) clarification of terms or concepts note readily understandable; (2) problem definition; (3) problem analysis using previous knowledge, (4) development of systematic inventory as from explanations obtained in step 3; (5) formulation of learning objectives and strategies for information acquisition; (6) information collection, individually; and (7) summary and test of new information ${ }^{1}$.

The application of new knowledge, especially the development of schemes or algorhythms used by experts to solve problems, has generated a new way to conduct POL sessions, which introduces the building of schemes or algorhythms as a means to knowledge search and problem-solving. The process may be summarized in 7 stages 16 :

1.Problem identification.

2.Development of a logic approach to the diagnostic process and identification of target learning subjects applicable to the development of an algorhythm aimed at solving the problem;

3. Individual information collection;

4.Derivation of an algorhythm to solve the problem using acquired information;

5.Application of the algorhythm for diagnostic clarity including history, physical and complementary tests;

6.Discussion of relevant data, activation of previous concepts, development of individual data interpretation, reorganization of such data and diagnostic conclusion;

7.Presentation and solution of different cases, representative of the same problem.

\section{PROBLEM FORMULATION}

Problems should be carefully developed aiming at adhering to learning goals, and should have the following characteristics: (1) be an objective description of a situation (intercurrence or disease) needing explanation of mechanisms, underlying processes or principles, and related to the program content; (2) allow for discussions, avoiding direct questions; (3) reflect situations which may be found in practice; (4) be compatible with residents' level of knowledge; (5) include most common intercurrences; (6) represent critical or urgent situations; (7) be associated to high morbidity or mortality rates;
(8) include problems known to be poorly handled or unknown by the community ${ }^{1}$.

Other authors ${ }^{17}$ use the Delphi technique, in which experts judge, define and give priority to characteristics, via consensus, to define most important characteristics of a problem. According to these authors, problems should (1) encourage thinking, analysis and reasoning; (2) assure self-oriented learning; (3) encourage the use of previous knowledge; (4) be proposed in actual contexts; (5) lead to the discovery of learning objectives; (6) arouse curiosity; (7) refer to subjects relevant to the specialty; (8) assure contextual amplitude through details which identify the situation; and (9) favor the use and acquisition of scientific vocabulary.

\section{ROLE OF TUTORS, STUDENTS AND INSTITUTIONS}

Tutors are learning facilitators in the POL process. So, the first change must be in the field of tutors who should acquire knowledge to be qualified for the function of tutors of student groups. Major difficulty found by tutors in teaching those groups is to be a facilitator and not a professor ${ }^{18}$. Tutors who are experts on the subject tend to be excessively directive and explain too much, preventing students to develop productive interactions during POL sessions ${ }^{19,20}$. The ignorance of group processes is another cause for trainers' failure since, in allowing the group to interact, group mechanisms and crises are easily unveiled. The ignorance of those mechanisms and their handling leads tutors to adopt directive and professorial behaviors, impairing the process of group learning ${ }^{21}$.

Students prefer trainers who master the subject, ask related questions, orient reasoning, engage with the group, give corrective feedback and do not inhibit students' spontaneity. This latter characteristic is primarily obtained by tutors' receptivity to students' opinions and statements, avoiding intimidating criticism ${ }^{22,23}$.

The implementation of a POL-based program must count on the commitment of tutors in the ideological field. Once this is obtained, the next step is a theoretical and practical qualification of trainers interested in participating in the program. Such qualification, in general achieved using the method itself, involves topics such as POL pedagogic and psychological bases, problem development, group mechanisms, evaluation methods, situational leadership, time management, dysfunctional groups diagnostic and handling, among others, according to the profile of the institutions or of their faculty members. It is important that trainers define, as they are exposed to training, additional contents meeting their own learning needs. These training sessions have been very positively evaluated by participants as to their applicability to the tutorial process ${ }^{16,24,27}$

The leadership style to be used by the tutor depends, basically, on the maturity of the group, which is manifested by the degree of confidence of its members, by their ability and motivation to accomplish their tasks and by the amount and type of feedback they need. Four leadership styles applicable to different groups were identified:

Revista Brasileira de Anestesiologia Vol. 53, N 2, Março - Abril, 2003 
1. Directive, used with immature groups with low ability and motivation to perform proposed tasks, emphasizes results with little emphasis to group's social sphere;

2. Educating, used with low to moderate maturity groups, is characterized by their willingness to learn in spite of their lack of capacity, emphasizes task understanding;

3. Motivating, used with moderate to high maturity groups, is characterized by unwillingness to perform tasks in spite of existing competence, emphasizes the participation of group members in the decision-making process as motivational strategy;

4. Delegating, used with highly mature groups, is characterized by competence and willingness to perform tasks, emphasizes group members empowerment and is characterized by less leader facilitation.

Each leadership style uses different powers to obtain results. The directive style uses the coercive power based on punishments; the educating style uses the power of rewarding good performance; the motivating style uses the referential power based on the identification of group members with the leader. In the delegating style, members perceive the leader as the most competent group member, and respect is the element of power guiding relationships with the leader. It is important to highlight that, in different situations, when facing different problems or pressed by external factors or internal conflicts, group members will adopt more or less mature behaviors, requiring the leader to be trained to recognize the level of group maturity depending on the moment, to adopt a more adequate leadership style not to impair the performance of the group ${ }^{26}$.

Students exposure to POL should be preceded by instructions about the method and its pedagogic justifications. Students should be informed about learning process dynamics, with notions about steps to be followed, types of evaluation and information sources, including trainers and their specific areas of interest so that they can be used as knowledge sources $^{29}$.

The institution is in charge of assuring comfort, safe environment and learning sources, such as libraries, Internet access and audiovisual material to be used by students. It is convenient to remind that POL sessions last 2 to 3 hours, so comfortable chairs, adequate lighting and air-conditioning are critical to avoid fatigue caused by discomfort, poor lighting and thermal inadequacy which contribute for a lower learning yield.

An important aspect of POL implementation is the discussion of the new curriculum with features meeting method's needs. New curriculum content taxonomies have been developed though a broad discussion among faculties, implementation of central curriculum committees or delegation of responsibilities to departments of the institution ${ }^{30-33}$.

Not all groups are productive. A dysfunctional group is characterized by lack of productivity. Most frequent manifestation of group dysfunction is the ritual behavior, which occurs when students maintain the false appearance of active involvement in solving proposed problems during POL sessions. This behavior is manifested (1) by the lack of connection bet- ween new ideas and previous knowledge base, (2) by not clearly defined learning objectives, or without specific relationship with the problem; and (3) by deficient individual study. As a consequence of this behavior, both the activation of previous knowledge and the elaboration of knowledge are absent, preventing learning process progress. Tutors not trained to identify and deal with this type of behavior tend to feel frustrated and try to remedy the lack of progress of the group by adopting the role of professors or by delegating it to a group member, that is, adopting the traditional education method. This attitude breaks POL philosophical basis and decreases group's self-esteem. Strategies proposed to solve this type of dysfunction suggest that the tutor: (1) poses more complex problems, requiring more interaction among group members; (2) poses questions activating previous knowledge retrieval and its application to solve the problem; (3) formulates, together with the students, clear and specific learning objectives; (4) encourages the expression of all group members and their feedback to colleagues; and (5) encourages students to apply new knowledge to other problems ${ }^{34}$.

Some group members may adopt the role of parasites and not actively participate in the job, having the other group members doing it for him/herself. These, in turn, in realizing this type of behavior, will contribute less and less to the activities of the group, characterizing the exit behavior. This way, group cohesion is broken and productivity drops. Another dysfunctional behavior is characterized by the excessively easy acceptance of explanations by group members, or by the inadequate explanation of learning topics ${ }^{35}$. In this situation, it is up to the tutor and the group to identify and discuss such behaviors, their repercussions on the learning process and alternatives to solve them.

Tutors may also contribute for group dysfunctions by adopting autocratic or excessively directive behaviors during POL sessions. In addition, on the other edge, tutors who are not committed to the work of the group (laissez-faire leaders or non-leaders) allow for the perpetuation of false concepts, impairing the learning process and decreasing group productivity ${ }^{28}$. A survey on students' perception about POL tutorial has revealed that students expect tutors to be skilled facilitators guiding them during learning and at the same time maintaining a positive group environment. Students do not want tutors to teach the program content because learning is perceived as their job 22,36,37.

The lack of feedback impairs the learning process for allowing the learning of false concepts and wrong problem solving strategies. The lack of corrective feedback contributes to problems transfer failure ${ }^{15}$. Feedback among group members should be encouraged, as well as individual feedback given to students by the tutor. Feedback should be corrective and encouraging and should be given to the group during all POL sessions (immediate feedback) ${ }^{38,39}$.

\section{IS POL BETTER THAN THE TRADITIONAL METHOD?}

There are no data on the effects of POL in Anesthesiology medical residency learning. Five extensive reviews comparing 
POL to traditional methods during the Medicine graduation course were performed from 1993 to 2000 using descriptive or meta-analytic methods. Several aspects were evaluated and the results are summarized below:

Albanese ${ }^{40}$, using the meta-analytic approach and a systematic review of existing studies to date has concluded that graduation students using the method had poorer performance in basic science tests and equal or better performance in clinical science tests, as compared to traditional program students. They used predominantly retrograde reasoning (deduction as from hypothetical solutions to explain observed data), had more versatile teaching strategies, more hours of study, and higher attendance to the library. As to the evaluation of students and tutors, authors have found a more positive perception of the learning environment, higher students' satisfaction and perception of being prepared although considering themselves deficient in basic sciences. As to obtaining first choice medical residency vacancies, POL students had similar percentages to traditional method. Although spending more time in POL sessions, tutors tended to believe that the method has more benefits and felt themselves satisfied. Authors also point to some restrictions and unsolved topics, such as the possibility of higher costs when groups have more than 40 students, slower knowledge acquisition rate, possibility of not adequately developing basic science knowledge and the possibility of graduating professionals unable to work by themselves.

Vernon ${ }^{41}$, using a meta-analytic approach, has concluded that (1) program evaluation results have favored POL; (2) there has been no difference between methods as to grades obtained in national tests; (3) POL students place more emphasis in understanding underlying processes while traditional method students tend to be more concerned with knowledge reproduction. POL students tend to be more creative in terms of knowledge acquisition sources and have better performance in clinical areas and worse performance in basic areas as compared to traditional method students.

Berkson ${ }^{42}$, in a systematic review, has concluded that POL is not distinguishable from traditional teaching, may be stressing for students and tutors and is excessively expensive for teaching institutions.

Thomas $^{43}$, in another systematic review, has concluded that POL students were not more motivated to study than traditional method students, that there has been a predominance of retrograde reasoning and that there were no differences between methods in the ability to structure knowledge in the clinical context. POL students, however, were more diversified in knowledge acquisition sources.

Colliver ${ }^{44}$, using a meta-analytic approach, has concluded that effects of previous meta-analysis and prospective studies are minor and statistically insignificant. He has also concluded that POL has a more challenging, motivating and agreeable approach to medical education, but its superiority as compared to traditional teaching is still to be proven. In his meta-analysis, the author suggests that there is no sound theoretical basis to justify POL. In this study, significance level was more than 0.8 standard deviation, much stricter crite- ria than those used in previous meta-analytic comparisons and the only theory considered was contextual learning ${ }^{14}$. There are three major POL advantages: higher level of students and professors satisfaction with the method, more diversification of knowledge reference sources and more time expended with individual study.

In the absence of POL disadvantages as compared to traditional methods, these reasons are in themselves enough to justify its use, taking into account that resident physicians and tutors satisfaction is a major dimension of medical residency quality ${ }^{45,46}$.

\section{REFERÊNCIAS - REFERENCES}

01. Schmidt HG - Problem-based learning: rationale and description. Med Educ, 1983;17:11-16.

02. Norman GR - Problem-solving skills, solving problems and problem-based learning. Med Educ, 1988;22:279-286.

03. Schmidt HG - Foundations of problem-based learning: some explanatory notes. Med Educ, 1993;27:422-432.

04. Barrows HS - A taxonomy of problem-based learning methods. Med Educ, 1986;20:481-486.

05. Bordage G - Elaborated knowledge: a key to successful diagnostic thinking. Acad Med, 1994;69:883-885.

06. Neame RLB, Powis DA - Toward independent learning: curricular design for assisting students to learn how to learn. J Med Educ, 1981;56:886-893.

07. Spencer JA, Jordena RK - Learner centered approaches in medical education. BMJ, 1999;318:1280-1283.

08. Schmidt HG - Assumptions underlying self-directed learning may be false. Med Educ, 2000;34:243-245.

09. Slotnick HB - How doctors learn: physicians' self-directed learning episodes. Acad Med, 1999;74:1106-1117.

10. Slotnick HB - Physicians' learning strategies. Chest, 2000;118:18S-23S.

11. Slotnick HB - How doctors learn: the role of clinical problems across the medical school-to-practice continuum. Acad Med, 1996;71:28-34.

12. Schmidt HG, Norman GR, Boshuizen HPA - A cognitive perspective on medical expertise: theory and implications. Acad Med, 1990;65:611-621.

13. Mandin H, Jones A, Woloschuk W et al - Helping students learn to think like experts when solving clinical problems. Acad Med, 1997;72:173-179.

14. Albanese M - Problem-based learning: why curricula are likely to show little effect on knowledge and clinical skills. Med Educ, 2000;34:729-738.

15. Norman GR, Schmidt HG - The psychological basis of problem-based learning: a review of the evidence. Acad Med, 1992;67:557-565.

16. Mandin $\mathrm{H}$, Harasym $\mathrm{P}$, Eagle $\mathrm{C}$ et al - Development of a "clinical presentation" curriculum at the University of Calgary. Acad Med, 1995;70:186-193.

17. Des Marchais JE - A Delphi technique to identify and evaluate criteria for construction of PBL problems. Med Educ, 1999;33:504-508.

18. Hay PJ, Katsikitis M - The 'expert' in problem-based and case-based learning: necessary or not? Med Educ, 2001;35:22-26.

19. Kaufman DM, Holmes DB - The relationship of tutors' content expertise to interventions and perceptions in a PBL medical curriculum. Med Educ, 1998;32:255-261. 
20. Olmesdahl PJ, Manning DM - Impact of training on PBL facilitators. Med Educ, 1999;33:753- 755

21. Pinchón-Rivière E - O Processo Grupal, $6^{\mathrm{a}}$ Ed, São Paulo, Martins Fontes, 2000;167-177.

22. Kaufman DM, Holmes DB - Tutoring in problem-based learning: perceptions of teachers and students. Med Educ, 1996;30:371-377.

23. Kaufman DM, Mann KV - Basic sciences in problem-based learning and conventional curricula: students' attitudes. Med Educ, 1997;31:177-180.

24. Lloyd-Jones G, Ellershaw J, Wilkinson $S$ et al - The use of multidisciplinary consensus groups in the planning phase of an integrated problem-based curriculum. Med Educ, 1998;32:278-282.

25. Finucane P, Nichols F, Gannon B et al - Recruiting problem-based learning (PBL) tutors for a PBL-based curriculum: the Flinders University experience. Med Educ, 2001;35:56-61.

26. Dolmans DHJM, Wolfhagen IHAP, Schmidt HG et al - A rating scale for tutor evaluation in a problem-based curriculum: validity and reliability. Med Educ, 1994;28:550-558.

27. Dolmans DHJM, Wolfhagen IHAP, van der Vleuten CPM Long-term stability of tutor performance. Acad Med, 1996;71: 1344-1347.

28. Ellis DG, Fisher BA - Small Group Decision Making, $4^{\text {th }}$ Ed, New York, MacGraw Hill, 1994;177-213.

29. Birgegård G, Lindquist U - Change in student attitudes to medical school after the introduction of problem-based learning in spite of low ratings. Med Educ, 1998;32:46-49.

30. Antepohl W, Herzig S - Problem-based learning versus lecture-based learning in a course of basic pharmacology: a controlled, randomized study. Med Educ, 1999;33:106-113.

31. O'Neill PA - The role of basic sciences in a problem-based learning clinical curriculum. Med Educ, 2000;34:608-613.

32. O'Neill PA, Metcalfe D, David TJ - The core content of the undergraduate curriculum in Manchester. Med Educ, 1999;33: 121-129.

33. O'Neill PA, Morris J, Baxter CM - Evaluation of an integrated curriculum using problem-based learning in a clinical environment: the Manchester experience. Med Educ, 2000;34:222-230.

34. Dolmans DHJM, Wolfhagen IHAP, van der Vleuten CPM et al Solving problems with group work in problem-based learning: hold on to the philosophy. Med Educ, 2001;35:884-889.

35. Dolmans DHJM, Wolfhagen IHAP, van der Vleuten CPM - Motivational and cognitive processes influencing tutorial groups. Acad Med, 1998;73:S22-S24

36. Caplow JA, Donaldson JF, Kardash C et al - Learning in a problem-based medical curriculum: students' conceptions. Med Educ, 1997;31:440-447.

37. Bligh J, Lloyd-Jones G, Smith G - Early effects of a new problem-based clinically oriented curriculum on students' perceptions of teaching. Med Educ, 2000;34:487-489.

38. Parikh A, McReelis K, Hodges B - Student feedback in problem based learning: a survey of 103 final year students across five Ontario medical schools. Med Educ, 2001;35:632-636.
39. Valle R, Petra I, Martínez-González A et al - Assessment of student performance in problem-based learning tutorial sessions. Med Educ, 1999;33:818-822.

40. Albanese M, Mitchell S - Problem-based learning a review of literature on its outcomes and implementation issues. Acad Med, 1993;68:52-81.

41. Vernon DTA, Blake RL - Does problem-based learning work? A meta-analysis of evaluative research. Acad Med, 1993;68: 550-563.

42. Berkson L-Problem-based learning have the expectations been met? Acad Med, 1993;68:S79-S88.

43. Thomas RE - Problem-based learning measurable outcomes. Med Educ, 1997;31:320-329.

44. Colliver JA - Effectiveness of problem-based learning curricula: research and theory. Acad Med, 2000;75:259-266.

45. Elliot RL, Juthani NV, Rubin EH et al - Quality in residency training: toward a broader multidimensional definition. Acad Med, 1996;71:243-247.

46. Norman GR, Schmidt HG - Effectiveness of problem-based learning curricula: theory, practice and paper darts. Med Educ, 2000;34:721-728.

\section{RESUMEN}

Oliveira Filho GR - Bases Teóricas para la Implementación del Aprendizaje Orientado por Problemas en la Práctica Médica en Anestesiología

Justificativa y Objetivos - El Aprendizaje Orientado por Problemas $(A O P)$ es un método de enseñanza cuyo objetivo primario es la acumulación de conceptos médicos en el contexto de problemas clínicos, que ha sido largamente empleado en la graduación médica desde los años sesenta. El $A O P$ es baseado en la teoría del procesamiento de información, segundo la cual, la adquisición de nuevos conocimientos es facilitada por la activación de conocimientos preexistentes sobre el asunto, por la similaridad entre los contextos de aprendizaje y aplicación del conocimiento y por la elaboración de la información. Otras teorías han sido utilizadas para justificar el uso del método $A O P$ en la enseñanza de estudiantes de Medicina y en otras áreas. El método AOP utiliza los conceptos aplicados al aprendizaje de adultos, los cuales se aplican a los episodios de aprendizaje de médicos.

Contenido - Este articulo describe el método AOP, sus bases teóricas y psicológicas, el papel de instructores y estudiantes en el proceso y sugestiones cuanto a su implementación.

Conclusiones - Comparado a la enseñanza tradicional, el AOP tiene como principales ventajas la mayor satisfacción de los estudiantes e instructores, la mayor diversificación de las fuentes de consulta y busca de conocimiento y el mayor tiempo despendido en el estudio individual. Una vez que el método no presenta desventajas, comparado al método tradicional, él puede ser considerado como una alternativa válida para la enseñanza de la Anestesiología en la Práctica Médica. 